\section{Espacios públicos y calidad de vida: Consideraciones interdisciplinarias}

Public spaces and quality of life: Interdisciplinary considerations

\author{
Matías DZIEKONSKY*, \\ MARÍA JosÉ RodRÍGueZ ${ }^{\star *}$, \\ CARLOS MUÑOZ ${ }^{\star \star *}$ \\ Karla HenRíqueZ ${ }^{* \star * *}$, \\ AMAYA PAVÉZ ${ }^{\star \star \star \star \star}$, \\ AlejandRA MuÑOZ ${ }^{\star \star * \star * *}$
}

Escuela de Arquitectura, Universidad de Santiago, Chile. Correo electrónico: matias.dziekonski@usach.cl

* Escuela de Psicología, Universidad de Santiago, Chile. Correo electrónico: mariajose.rodriguez.a@usach.cl

.** Escuela de Arquitectura, Universidad de Santiago, Chile. Correo electrónico: carlos.munoz.p@usach.cl

.... Escuela de Psicología, Universidad de Santiago, Chile. Correo electrónico: karla.henriquez.@usach.cl

....* Facultad de Ciencias Médicas, Universidad de Santiago, Chile. Correo electrónico: amaya.pavez@usach.cl

......* Escuela de Psicología Universidad de Santiago, Chile. Correo electrónico: almunozt@gmail.com

\section{Resumen}

El crecimiento de las ciudades, específicamente en Chile, no ha traído aparejada, necesariamente, una mejora en la calidad de vida y en la salud mental de sus habitantes. Abordamos la conjunción entre espacios públicos, relaciones interpersonales y calidad de vida, pues no ha sido suficientemente tratada. La calidad de vida se encuentra estrechamente vinculada con las relaciones sociales, interpersonales, y con la integración social, las cuales se han visto afectadas por el desarrollo de la urbe. Se elabora un diálogo interdisciplinario entre la arquitectura, la psicología social y la antropología, en torno a los espacios públicos, la urbanización y los procesos de encuentro social, pues los espacios públicos son escenarios relevantes en lo urbano, que cumplen funciones en las relaciones interpersonales, la integración social y la calidad de vida. Resalta la necesidad de identificar criterios para el diseño de espacios públicos como espacios de encuentro, interacción social y vida ciudadana.

Palabras clave: espacios públicos, calidad de vida, relaciones interpersonales, integración social, interdisciplinariedad.

\section{Abstract}

The growth of cities, specifically in Chile, has not necessarily brought with it an improvement in quality of life and in relation to the mental health of the inhabitants. We address the conjunction of public spaces, interpersonal relationships, and quality of life, as it has not been sufficiently considered. Quality of life is closely linked with social and interpersonal relationships and with social integration, and these have been affected 
by the development of the city. We need an interdisciplinary dialogue between architecture, social psychology and anthropology around public spaces, urbanization and social encounters, as public spaces are relevant in urban settings, which perform functions in interpersonal relationships, social inclusion and quality of life. The need to identify criteria for the design of public spaces as places for meeting, social interaction, and civic life are highlighted.

Key words: public spaces, quality of life, interpersonal relationships, social integration, interdisciplinarity.

\section{Introducción}

Pretender escribir sobre el espacio público hoy, resulta ser, cada vez más, una ardua tarea. Aumenta la complejidad de la experiencia de lo público, dados los múltiples significados que se desprenden desde la polisemia del concepto -como su acepción política, estética, cotidiana y de las relaciones sociales, entre otras-, lo que es una expresión de ello.

Es por eso que hacemos un abordaje interdisciplinar, desde la arquitectura, la psicología y la antropología. La arquitectura aborda lo público como un proceso que va de la concepción a la construcción de un soporte espacial de lugares y espacios socialmente compartidos, para permitir que los miembros de una sociedad se encuentren e interactúen".

Los términos lugar y espacio, usados de manera indiferenciada en el lenguaje coloquial, son conceptos diferentes. En este artículo entenderemos que lugar está asociado con experiencias y vivencias, cuestión única para el individuo que las vive, mientras que espacio dice relación, más bien, con una percepción física -abstracta y geométrica- posible de definir, medir y cuantificar.
Desde la psicología social nos aproximamos a la comprensión del sujeto, como individuo y en su condición social, a través de su interacción con otras personas en un contexto determinado. A partir de la antropología interpretamos las convergencias y las divergencias entre la propuesta social del espacio público, y el uso y el sentido que esto tiene para las personas y los grupos desde una perspectiva cultural.

La articulación de estas tres disciplinas nos permitirá desarrollar una mirada transversal, donde se integren el espacio público y los seres humanos, analizados no sectorialmente como suele hacerse. Esto es así, pues consideramos que toda persona ${ }^{2}$ busca una armonía como individuo y como parte de un grupo, respecto del ambiente que lo rodea.

Por lo tanto, concebimos el espacio público como aquél que permite la construcción de identidades y de realidades individuales compartidas, que favorecen e influyen en la integración social. Es así que el espacio público, representado en la plaza, es indispensable como lugar para establecer relaciones entre las personas y favorecer la asociatividad, dotándolas -en ese proceso- de elementos identitarios compartidos, y construyendo su ciudadanía al reforzar su rol activo en dicha participación. Por ello es que la plaza -en tanto esencia del espacio público- nos rememora el ágora griega que, al ser el espacio público por antonomasia, permite la interrelación social y su natural derivación en la vida política. Así, en ese espacio tangible, se expresa, contrasta y desarrolla el universo simbólico de los distintos actores que se encuentren en él.

\footnotetext{
Para efectos de este artículo usaremos las expresiones de individuo, sujeto y persona de manera indistinta, a pesar de que a nivel ontológico existen diferencias.
} 
En primer lugar, parece importante reflexionar respecto del espacio público de la plaza, cuándo y cómo emerge, y cuál es el sentido que este constructo cultural trasunta hasta la actualidad. En segundo lugar, podremos hacernos algunas preguntas sobre este tipo de espacio público en el contexto de la modernidad en Chile:

¿De qué manera la configuración de los espacios públicos está influyendo en la vida de las personas, en su salud mental, en sus relaciones con otros, en su vida ciudadana, en su calidad de vida?

¿Está el diseño ${ }^{3}$ de los espacios públicos respondiendo a la necesidad de una mejor calidad de vida e integración social de las personas y su desarrollo?

¿En qué medida el diseño urbano promueve un encuentro interpersonal, la comunicación entre ciudadanos, la vida en comunidad, tan necesarias para la salud de las personas?

¿Cómo podemos potenciar el diseño de los espacios públicos de modo tal que favorezca la calidad de vida de sus usuarios?

A raíz de estas preguntas, nos percatamos de la necesidad de mirar el espacio público desde la interdisciplinariedad, integrando las perspectivas de la psicología, de la antropología y de la arquitectura -paisajismo mediante-, para así avanzar en la creación de una metodología de diseño de espacios públicos, que sea capaz de acoger las diversas necesidades de los habitantes urbanos. El producto de esta reflexión y discusión se presenta a continuación.

Entendemos, por nuestro trabajo académico y profesional, que el diseño es un proceso que se inicia como una formulación y termina como una formalización, teniendo tres fases: una primera fase "conceptual", que articula una narración que lo fundamenta; una segunda fase "proyectual", que se expresa en imágenes, planos y especificaciones, y; una tercera fase "operacional", cuyo propósito es materializarlo.

\section{Espacios públicos, urbanización y socialización}

Una primera aproximación nos hace pensar que el espacio público, tal como lo entendemos hoy, aparece como tal con el advenimiento de la vida humana en las ciudades, es decir, ciudad y espacio público emergen probablemente en la historia, de forma simultánea. Sin embargo, es dable pensar que en formas de vida no urbana y en formas de vida pre o proto urbanas, también fue posible distinguir "lugares" o "espacios públicos" que los grupos humanos reconocieran como tales. Estos espacios tenían ciertas características que los hacían aptos para ciertas actividades que allí se realizaban, esporádica o periódicamente, fueran éstas religiosas, cívicas, militares o comerciales, y quienes los usaban asumían que no era posible apropiárselos, pudiendo o debiendo ser compartidos, probablemente por sus características o por las posibilidades que ofrecían.

Ahora bien, la ciudad como fenómeno emergió en el contexto de la evolución humana, asociada con el desarrollo de nuestras capacidades para procurarnos el necesario sustento. Este cambio, acaecido hace más de diez mil años atrás, consistió en que nuestra especie iniciaba un proceso de sedentarización, alejándose paulatinamente del nomadismo, fenómeno que le permitió pasar de una relación básicamente reactiva con la naturaleza, a una relación proactiva con ella: "[e]s el momento en que la agricultura y la ganadería sustituyen a la caza como sistema básico de supervivencia de nuestra especie" (Morris 2000: 11).

Al interior de estos asentamientos, un proceso cultural y político habría de vivirse desde esos 
días donde los liderazgos se organizaron en torno a un poder centralizador -un rey y su palacio legitimados divinamente, cuestión que duraría hasta que su decadencia fue toleradaextendiéndose hasta el día de hoy. Emergerían nuevas formas políticas donde las familias aristocráticas -con el tiempo los clanes, los grupos organizados en torno a intereses comunes- disputarían el poder según el principio de una lucha civilizada (y a veces no tanto), por la vía de una justa oratoria en la plaza pública.

Es en el espacio abierto de la ciudad que los pretendientes al poder se confrontarían y se elegiría al primus inter pares -al primero entre iguales- para ejercer un liderazgo que sería obedecido voluntariamente, debido a leyes consensuadas y no a la voluntad tiránica y arbitraria de un hombre. Esta práctica inauguraría el advenimiento de la ciudadanía (civitas) y de la ciudad, que dejaría de ser una mera aglomeración orgánica adosada a una centralidad fortificada de la que depende, y se transformaría en un hecho histórico notable, pues es el uso de la palabra en el debate público el que configuraría la polis: una comunidad política administrada por sí misma, y constituida generalmente por una agrupación urbana y un territorio circundante.

Sobrevendría el debate público en un espacio público, en la plaza, en el ágora, lugar de excelencia de los intercambios, de la comunicación y del lenguaje, donde éste lograría "fijar el sentido de las cosas y hacer circular y evolucionar ese sentido sin que él encierre contradicciones" (Gagin 2002: 98). Así, esta menor dependencia de la naturaleza plantearía desafíos respecto de los excedentes que comenzaron a generarse, cuestión que se resolvería creando ciudades para asentarse, murallas para protegerlos y protegerse, alfabetos, dinero y sistemas contables para comunicarlos, intercambiarlos, dimensionarlos y evaluarlos, y por último, liderazgos que se transformaron en dinastías jerárquicamente estructuradas para controlarlos, defenderlos y ampliarlos.

Con ello la humanidad realizaba una revolución, un cambio sustancial en su modo de relacionarse entre sí y con el mundo. Es lo que algunos autores denominan como la "primera ola de cambio" (Toffler y Toffler 1995: 25), la cual estableció fundamentales ventajas, pero no estuvo libre de desafíos. "No pasaría mucho tiempo antes de que los almacenes de alimentos propiciaran el crecimiento de los pequeños asentamientos hasta formar grandes pueblos, que pronto derivaron en núcleos poblacionales mercantiles que a su vez dieron paso a enormes ciudades" (Morris 2000: 13).

Todo este complejo proceso, no exento de avances y retrocesos, ha marcado un hito en años recientes. La población urbana del mundo en su conjunto, según cálculos del Fondo de Población de las Naciones Unidas, alcanzó en el año 2008 el 50\%, equilibrándose ambos grupos de población, la urbana y la rural, en una cifra en torno a los 3.300 y 3.400 millones cada una (Obaid 2008). A partir de esa fecha, la población urbana ha ido incrementando sostenidamente.

Si pensamos que hace cien años, " 2 de cada 10 personas en el planeta vivían en una zona urbana y que en el año 2050, 7 de cada 10 personas vivirán en una ciudad", podemos concluir que este incremento de residentes urbanos -que hoy "está creciendo a razón de casi 60 millones cada año"- generará a futuro importantísimos desafíos para nuestra especie. 
Esto es así dado que "casi todo el crecimiento de la población urbana en los próximos 30 años se producirá en las ciudades de los países en desarrollo" (Global Health Observatory 2013: en línea).

Si ponemos estas cifras en perspectiva, veremos que en un período que podríamos estimar de entre unos 10.000 a 15.000 años, hemos cambiado radicalmente de forma de vida. Este tiempo, mirado desde la teoría del origen de los humanos modernos -que asume un tiempo de entre 140.000 y 200.000 mil añoses una cifra que se sitúa entre un $5 \%$ y un $10 \%$ del tiempo de nuestra existencia (Mayell 2010).

Visto así, el cambio es inmenso, pues nuestra especie se ha aglutinado en el espacio geográfico vía proceso de urbanización, lo que ha implicado una concentración y una densificación en las ciudades, aumentando la cantidad de personas por unidad de superficie, que debe ser conciliada con fenómenos de sustentabilidad, por una parte, y con fenómenos culturales y psicológico-adaptativos, por otra. No es casual, entonces, que el tema del espacio público sea foco de reflexión, investigación y acción, considerando el dramático proceso de urbanización de los últimos 200 años, que ha devenido en una aglomeración humana sin precedentes en las grandes urbes.

En apenas diez mil años las rutilantes luces de la civilización moderna habían emergido de la oscuridad de la primitiva existencia tribal. El ser humano no había tenido tiempo de evolucionar hacia una nueva especie de animal urbano. Biológicamente, los habitantes de las metrópolis, con su elegante indumentaria, sus coches rápidos y sus viviendas aisladas, son idénticos a los pobladores de la Edad de Piedra que pintaban escenas cinegéticas en las paredes de las cavernas. Tienen la misma anatomía, y actúan movidos por las mismas necesidades. Lo único que ha cambiado es su estilo de vida. Un cambio espectacular por donde se lo mire (Morris 2000: 13).

Estas afirmaciones nos informan sobre la compleja deriva evolutiva de nuestra especie $\mathrm{y}$, de paso, nos señalan la relevancia que tiene el proceso de socialización sobre nosotros; proceso que prepara y moldea las formas de interacción que tenemos, y que nos permite enfrentar la vida de manera colectiva. A través de la socialización, que se da por medio de la interacción social (Berger y Luckmann 2001), aprendemos normas, valores, formas de comportamiento y categorías para percibir la realidad.

Las experiencias que el ser humano va teniendo, inducidas normalmente por agentes sociales ${ }^{4}$-como son los miembros de la familia, las instituciones educativas, los pares y los medios de comunicación a los que se tenga acceso- serán determinantes en la percepción e interpretación que la persona desarrolle de su entorno, así como en su comportamiento futuro respecto de él y en él. Los espacios públicos, lugares donde se dan contactos e intercambios con otros, juegan también un rol crucial en las percepciones, interpretaciones y acciones que se asuman en ellos. Se podría afirmar que es en el espacio público donde se concreta el contrato social, y donde las personas se transforman en seres sociales: " $[\mathrm{e}]$ ncontrar una forma de asociación que defienda y proteja con toda la fuerza común la persona y los bienes de cada asociado, y en la cual cada uno, uniéndose a todos, aún puede obedecerse

\footnotetext{
Ser agente, o la capacidad de agencia, es la capacidad de generar acciones que impliquen cambios sociales. Este concepto permite reconocer que el individuo no es un mero producto de su entorno, sino que también construye y transforma realidades.
} 
a sí mismo y permanece tan libre como antes" (Rousseau 1980: 19).

Los efectos a los que nos expone la vida urbana contemporánea, y su futuro incremento, nos hacen reflexionar sobre la necesidad de comprender los diferentes elementos que la integran. El espacio público juega un rol en la necesaria socialización y en la calidad de las relaciones interpersonales que podamos -o notener en él. El arreglo físico-espacial, su diseño, no determina totalmente el comportamiento humano, pero sí cataliza o inhibe conductas latentes en las personas (Fuentes y Lawner 2013). Así, el espacio público tiene finalmente implicancias en la calidad de vida de todos nosotros, y éste es el tema que necesitamos abordar, atendiendo su complejidad.

\section{Un problema contemporáneo}

Junto al crecimiento de los asentamientos humanos que dejaron de ser polis para ser urbe (Neira 1997), podemos cotejar un incremento del malestar en la población, y el aumento de las tasas de depresión, violencia, soledad y suicidios ${ }^{5}$. Dado que lo urbano se ha configurado como el gran escenario de la vida social tanto en Chile como en el mundo, y que, como sugiere la evidencia, a futuro esto se incrementará6, nos resulta pertinente abordar el desafío de prevenir el detrimento de la salud y la calidad de vida, y potenciar el sano desarrollo de la misma desde el diseño de las ciudades, en

Cuya tasa en Chile supera en promedio a la de los países de la OCDE (Valdés y Errázuriz 2012).

6 Según el último Censo Nacional del año 2012, en los últimos 20 años la población de las capitales regionales creció un $20 \%$, en tanto las respectivas regiones lo hicieron en un 13\% (Orellana 2013). especial desde los espacios públicos, donde, por excelencia, se despliega la vida ciudadana, considerando para ello las características del espacio y de la población que los utiliza.

En este sentido, la psicología social-ambiental estudia las interrelaciones entre las personas y el medio ambiente, considerando este último como el entorno físico y social en el que los individuos y las colectividades se desenvuelven y del cual forman parte. A partir de sus aportes, podemos comprender que el medio ambiente no sólo es considerado como un escenario o lugar -un soporte para la acción humana, como podría considerarlo la arquitectura y el paisajismo-, sino que debe entenderse como el contexto en el que se dan las interacciones sociales, donde el fenómeno psicológico puede estudiarse a partir de la relación con el medio sociocultural, espacial y temporal en donde sucede (Botero 2009).

De esta manera, podemos decir que toda relación se da en un contexto particular o locus. El término locus, de raíz latina, dice relación con "el lugar". Supone que los lugares son constructores de la subjetividad de los individuos y grupos. Edward S. Casey lo señala claramente cuando dice que "cualquiera sea la verdad para el espacio y el tiempo, esto es mucho más cierto para el lugar: estamos siempre inmersos en él y no podemos hacer nada fuera de él" (1998: ix).

La psicología ambiental considera respecto del estudio del lugar que éste posee un significado propio, que puede surgir desde las características físico-estructurales, de la funcionalidad que le otorga a las prácticas sociales, de las interacciones que se producen entre las personas a nivel simbólico o desde 
los niveles individuales -que le otorgan un significado personal y subjetivo, o social-, dotando al significado de elementos construidos mediante las intersubjetividades (Valera 1996). Paralelamente, desde el construccionismo social tenemos consciencia de que las personas pueden elaborar construcciones simbólicas diferentes sobre lo que consideran-y constituyesu calidad de vida, así como sus imaginarios de felicidad ${ }^{7}$ y buena vida (Rodríguez 2012), y que éstas son construidas también desde la interrelación con "el lugar" o los lugares donde las personas se desenvuelven.

A partir de lo anterior, podemos señalar que las personas transforman los lugares que son parte de su vida cotidiana y, a su vez, éstos transforman a las personas, existiendo una relación dialéctica entre ambos. De esta forma, la significación y la interpretación elaboradas en relación con los espacios públicos y sus usos podrían ser diferentes dependiendo de variables culturales y socio-demográficas.

A ello sumamos lo que señala el interaccionismo simbólico, a saber, que las personas se vinculan con los objetos -en este caso el espacio- a partir de lo que representan para él (Valera 1996). Por todo esto, es necesario mirar el fenómeno en su complejidad, y para lograrlo, incorporar la subjetividad es crucial, ya que nos permite sumar al interés por la calidad de vida el estudio del espacio público y las relaciones interpersonales como constructos dinámicos y procesos de alta complejidad.

Lo público puede ser el espacio de lo propio o de lo ajeno. $Y$ es que las relaciones

Felicidad e infelicidad son comprendidas en este trabajo como significantes que aluden en el lenguaje de la vida cotidiana a sentimientos de bienestar o malestar. interpersonales en los espacios públicos van a estar mediatizadas tanto por las experiencias previas al uso de lugares de este tipo, como por las creencias asociadas a dicho uso y a las funciones atribuidas al espacio. Del mismo modo, este uso estará mediado por distintos significados, y también por las representaciones sociales que inducirán a los sujetos a acercarse o alejarse de ciertos tipos de lugares. Ello se puede relacionar con una cierta tipología de espacios públicos, pudiendo ser éstos de vinculación, recreación, congregación, entre otros.

Además, relacionarse con otras personas en espacios públicos implica demostrar los intereses personales y los tipos de interacciones que se establecen con otros. Es, pues, un escenario donde el sujeto está permanentemente expuesto. Es indicar, de alguna manera, las propias creencias y emociones. En este contexto, resulta necesario proponer que las significaciones de los espacios públicos y su asociación con las relaciones interpersonales se afectan en el devenir histórico de una sociedad. Sin duda, en el caso de Chile, estas interacciones y usos pueden haberse visto afectadas por los innumerables cambios estructurales y culturales producidos desde su fundación.

Creemos importante investigar la relación entre espacios públicos, relaciones interpersonales y calidad de vida, pues consideramos que esta intersección no ha sido suficientemente profundizada al tratar los problemas de malestar social y salud mental en nuestro país. Interesa comprender cómo y en qué medida los espacios públicos están cumpliendo funciones clave, para orientarnos a la hora de planificar acciones que busquen mejorar las relaciones 
interpersonales, la integración social, la salud $y$, por ende, la calidad de vida de los habitantes de las ciudades. Amerita identificar y evaluar los criterios para el diseño de espacios públicos que han demostrado ser relevantes a la hora de mejorar la calidad de vida de las personas, sobre todo en lo que respecta a su papel como espacios de encuentro, interacción social y vida ciudadana.

Resulta necesario incorporar al diseño y desarrollo de los espacios públicos la mirada de la subjetividad y los significados, en relación con los espacios que las personas utilizan, así como la dimensión de la vida social-interpersonal tan determinante del bienestar humano (Javaloy, Páez y Rodríguez 2008). De esta manera, se podría comprender mejor aún cuáles son las buenas y malas prácticas referidas al diseño de los espacios públicos. Este conocimiento puede ser relevante en la planificación de acciones para mejorar la calidad de vida de los habitantes de las ciudades.

Para la construcción de una sociedad inclusiva y democrática, que favorezca el desarrollo y bienestar de las personas, es importante que en la planificación de los espacios públicos se considere a todos los miembros de la comunidad y, así, integrar a las personas desde sus individualidades (considerando sus edades, capacidades diferentes, estilos de vida, intereses etc.). De este modo, se fomentan las interrelaciones entre el espacio, las personas y su entorno, permitiendo la flexibilidad de usos, adecuando el espacio a las necesidades en cada una de las etapas del desarrollo del ser humano, y también entendiendo que los espacios, a partir de la configuración de sus infraestructuras, inciden en la manera en que se establecen las relaciones entre las personas.
Para esto es necesario promover, mediante una política integral, un sistema habitacional vivienda-barrio-ciudad, en el cual colaboren la arquitectura, el desarrollo urbano, la demografía, la sociología, la psicología social y ambiental, la geografía, la salud y el medio ambiente, entre otras disciplinas. Desde aquí sería posible definir los fenómenos que permitirán proyectar la generación de una ciudad inclusiva (Vázquez-Honorato y SalazarMartínez 2010). Esto es así pues sólo una mirada interdisciplinar nos permitirá abordar la complejidad de la vida urbana, con el horizonte ético de contribuir a la vida de las personas en el contexto de la sociedad.

Además, es prioritario para la sostenibilidad social propiciar la búsqueda de incentivos espaciales que faciliten el desarrollo de actividades entre las familias, y las posibilidades de multiplicidad de usos que enriquezcan el desarrollo social. También es necesario que estimulen el aprendizaje del desarrollo ambiental y físico, la posibilidad de ambientes armónicos, la reconstrucción del tejido social, y la conformación de una sociedad más cohesionada, capaz de incentivar las relaciones y los apoyos entre individuos en la búsqueda de soluciones a sus problemas cotidianos, definiendo con ello una cohesión social estable (Vázquez-Honorato y Salazar-Martínez 2010).

\section{Calidad de vida, relaciones interpersonales e integración social en el espacio público}

La salud es un estado de completo bienestar físico, mental y social, y no solamente la ausencia de afecciones o enfermedades.

Organización Mundial de la Salud (1948) 
La definición de salud adoptada por la Conferencia Sanitaria Internacional, celebrada en Nueva York en 1946 y firmada por los representantes de 61 Estados (World Health Organization 2013: en línea), entró en vigor el 7 de abril de 1948. Esta enunciación, a pesar de los cuestionamientos a su formulación, no ha sido modificada hasta la fecha. Independientemente de las discusiones que en torno suyo puedan existir, lo cierto es que podríamos afirmar que queda un arduo camino que recorrer para lograr el estado de completo bienestar físico, mental y social. Lo que nos queda claro es que el horizonte de la salud plena es una configuración que comprende el desarrollo del potencial tanto físico, como psicológico y social, cuando menos.

En este sentido, resulta apropiada la noción de calidad de vida como un constructo multidimensional para aproximarnos a la comprensión del bienestar humano. Felce y Perry (Cit. en Barrientos 2005) definen la calidad de vida como el bienestar general de las personas. Dicho bienestar comprende descriptores objetivos y evaluaciones subjetivas, relativas al bienestar físico, material, social y emocional, junto con el desarrollo personal y de actividades, todos ellos mediados por los valores, las aspiraciones y las expectativaspersonales. Mientras los descriptores objetivos constituyen las condiciones de vida, la evaluación subjetiva refiere a las percepciones y al sentimiento de las personas.

La radiografía chilena respecto de la calidad de vida, en general, deja entrever problemáticas en el bienestar psicológico -o salud mental-, así como en el bienestar subjetivo -o autopercepción de bienestar- de las personas ${ }^{8}$. Según la

Encuesta Nacional de Salud Chile, 2009-2010; Encuesta Nacional de Calidad de Vida y Salud, 2006 (Valdés y Errázuriz 2012).
Organización para la Cooperación y el Desarrollo Económico (2013), Chile debe prestar atención a una serie de índices obtenidos en indicadores de corte tanto subjetivo como objetivo. Al hacer un ranking -considerando todos los indicadoresChile ocupa el lugar $\mathrm{N}^{\circ} 33$ de un total de 36 países evaluados. En el ítem relativo a la calidad del medio ambiente en que se vive, Chile ocupa el último lugar. Respecto a los componentes subjetivos de la calidad de vida, el informe para Chile del Programa de Naciones Unidas para el Desarrollo (2012) deja en evidencia la relación entre las experiencias de soledad, infelicidad, estados de ánimo negativos y síntomas depresivos, siendo la soledad una de las manifestaciones más significativas del malestar subjetivo individual, la cual está positivamente relacionada con el nivel socioeconómico ${ }^{9}$.

Y es que la calidad de vida, sobre todo en su dimensión subjetiva, tanto social como individual, se vincula estrechamente con las relaciones sociales e interpersonales, que son una fuente incesante de placer, afecto y reconocimiento, además de cuidado y satisfacción de necesidades alimentarias y de seguridad. Desde el punto de vista psicológico, el individuo va satisfaciendo muchas de sus necesidades en la medida en que pertenece y forma parte de grupos humanos. Para lograrlo, la persona responde al comportamiento que los demás -sus padres, pares, conciudadanos- van modelando en su proceso de crecimiento. De esta manera, en las relaciones interpersonales la sociedad reproduce y mantiene un cierto orden social. Es por esto que una rica vida interpersonal es necesaria no sólo para la persona y su calidad de vida individual, sino

\footnotetext{
Las personas de los grupos D y $E$ dicen sentirse con mayor frecuencia en soledad, en comparación con las personas de las de clases socioeconómicas más altas.
} 
también para la sobrevivencia de la especie humana (Javaloy, Páez y Rodríguez 2008).

En cuanto al papel de las relaciones interpersonales en la calidad de vida, se ha detectado que el descenso del contacto social disminuye el bienestar e, inversamente, si éste se incrementa el bienestar mejora. La soledad y la ausencia de otros para compartir las experiencias generan profundos malestares. De acuerdo a las investigaciones, quienes se consideran a sí mismos como muy solitarios o con escasos contactos sociales, también reconocen sentirse deprimidos o muy infelices (Cit. en Javaloy, Páez y Rodríguez 2008).

A la soledad afectiva se adhiere el sentimiento de sentirse solo, es decir, el aislamiento psicológico, sumado a un déficit en las relaciones sociales, que según Weiss (Cit. en De Jong Gierveld y Van Tilburg 2006), puede materializarse como soledad social -no tener amigos o conocidos con quienes compartir intereses y actividades-, y/o como soledad emocional -ausencia de un vínculo profundo e íntimo con otra persona-. Cuando la soledad se ha vuelto crónica se dan conductas como llorar, dormir, tomar tranquilizantes, ver la televisión sin objetivo fijo, buscar refugio en un trabajo absorbente o en el consumo de alcohol y drogas.

En suma, la falta de integración o el aislamiento social sehaasociadoalaansiedady ladepresión, así como a mayor mortalidad y morbilidad, a peor recuperación de enfermedades, a una mayor reactividad al estrés, y a formas de afrontamiento menos adaptativas (Cit. en Javaloy, Páez y Rodríguez 2008). En cambio, vivir integrado interpersonalmente y socialmente es importante para la calidad de vida y la salud mental, y genera una serie de espirales sociales virtuosas que son necesarias para el medio social y el ambiente en general. Las redes sociales, aparte de ser un soporte afectivo, conforman un entramado de apoyo que permite satisfacer ciertas demandas cotidianas y, a la vez, abren cabida a nuevas oportunidades de desarrollo de personas e instituciones sociales como la familia, la amistad, los grupos de apoyo, las acciones altruistas y pro-sociales, la acción ciudadana y la integración comunitaria y barrial.

No obstante, en la actualidad, el escenario pareciera no generar las condiciones que facilitan estos procesos. La movilidad de la población muchas veces implica cortar lazos con familiares y amigos, las relaciones secundarias que se establecen en la gran ciudad son más formales, frías y poco íntimas, y se ha derivado a una disminución de las relaciones con la familia amplia. Todo lo anterior, sumado al incremento de divorcios y al auge de valores culturales individualistas como son la competitividad y la búsqueda del éxito personal-, ha jugado en contra de una rica vida interpersonal (Cit. en Javaloy, Páez y Rodríguez 2008).

Para superar el malestar es necesario, entonces, configurar un contexto social que facilite las relaciones interpersonales y la integración en lugar de la exclusión social. Como lo señalan Vázquez-Honorato y Salazar-Martínez,

necesitamos lograr una integración comunitaria que enriquezca el desarrollo social en ambientes armónicos que reconstruyan el tejido social, incentiven las relaciones y los apoyos entre los individuos, en la búsqueda de soluciones a sus problemas cotidianos y ofreciendo con ello una mejor cohesión social (2010: 12). 
Consideramos que el espacio público es un escenario crucial para el desarrollo de estas interacciones, intercambios y relaciones humanas. Sin embargo, de acuerdo a lo señalado por Valera (Cit. en Fernández-Ramírez y Vidal 2008), en el espacio público no es posible ejercer un control directo y efectivo de la interacción con los demás, a diferencia de lo que acontece en el espacio privado, donde las estrategias de regulación de la interacción son más variadas y eficaces.

Lo anterior se relaciona con lo planteado por Salcedo y Caicedo (2008), en cuanto al temor que pueden tener las personas hacia el espacio público, lo que se puede vincular con el temor al otro existente en las culturas humanas. En este sentido, lo desconocido -tanto el espacio público como las personas que circulan en éste- impide al sujeto desenvolverse de manera activa, lo cual puede potenciarse por dificultades para entender las claves ambientales, como, por ejemplo, su diseño, las señales y/o el mobiliario urbano.

En este sentido, la seguridad ha sido una problemática significativa para Chile. Los habitantes reclaman escasez de protección frente a crímenes, delitos y hurtos. Según el Balance de la Delincuencia (Fundación Paz Ciudadana 2013), el porcentaje de delitos en la vía pública se ha mantenido entre 2010 y 2012 en un $43 \%$, y la percepción de inseguridad da cuenta de que el $47,7 \%$ de los chilenos se siente inseguro en zonas públicas. Este temor está asociado a zonas urbanas, lugares que suelen tratarse como inherentemente inseguros (Programa de las Naciones Unidad para el Desarrollo 2012). Este fenómeno es incidido por múltiples variables, no pudiéndose descontar las relativas a las condiciones y características de los espacios públicos, dado que existen condiciones espaciales -tales como la iluminación y la visibilidad, sólo para citar dos de ellas- que favorecen o inhiben la ocurrencia de delitos.

Lo anterior se puede analizar a partir de lo que Candia (2001) señala en relación con la conceptualización de la ciudad, en tanto espacio físico desde el cual -y para el cualse pensaba e interactuaba en los antiguos poblados de menor escala. En este contexto, la sensación de inseguridad era menor, debido a que mientras fuese posible percibirse $y$ comunicarse cara a cara con el otro, se podía confiar en él ${ }^{10}$. No obstante, dicha confianza se ha ido perdiendo en la medida en que ha aumentado la distancia entre el espacio de lo público y el espacio de lo privado. Los otros, imperceptibles y desconocidos, devienen no confiables, y al ser personas no confiables, comienzan a ser distinguidos desde el temor, vistos como peligrosos. Esto constituye un ejemplo más de cómo los usos del espacio público son sensibles a las dinámicas históricas y sociales de cualquier colectivo humano.

\section{El espacio público y la integración de la vida social}

A partir de una definición contemporánea, el espacio público puede caracterizarse como aquél en que cualquier persona tiene el derecho de estar, en oposición a los espacios privados. En estos últimos, la estadía está permitida

La concentración de personas y la búsqueda de formas de colaboración ha sido parte de la experiencia humana desde tiempos prehistóricos. Entonces, como ahora, la gente se reunía en busca de protección, de convivencia y de intercambio (Laboratorio BMW Guggenheim 2013). 
básicamente por la relación de propiedad, pudiendo ser ésta privada, perteneciente a una persona o a alguna institución social, ya sea comunal, gubernamental, militar u otra.

Legalmente, el espacio público moderno nace al separar formalmente la propiedad urbana privada de la pública, lo que involucra reservar, desde el ordenamiento territorial y urbano, una cantidad de suelo disponible para los usos y las necesidades sociales propias de la vida urbana, tales como las actividades requeridas por el transporte -en sus diferentes formas-, como las actividades productivas, comerciales y culturales, dicho sintéticamente. Esto implica que el espacio público está sometido a regulaciones específicas por parte de la administración pública, sea ésta la estatal central, comunal local y/u otras.

Si bien la plaza fue, probablemente por muchos siglos, el arquetipo del espacio público, la época moderna amplía nuestra oferta de espacios posibles ofreciéndonos el parque público, un área de espacio abierto dispuesta para el uso recreativo colectivo, pudiendo estar en un estado natural o no, reservada para el disfrute humano o para la protección de la vida silvestre y el hábitat natural. Su origen se remonta al Medioevo europeo: tierras reservadas para la caza de la realeza y la aristocracia, situadas alrededor de mansiones y casas de campo, proclamando la riqueza y el estatus del propietario, desarrollando diversas estéticas, y dando origen al diseño del paisaje. El crecimiento de las ciudades fue dando pie a que algunos de ellas terminaran convertidas en lugares para el público, como nuestro Parque Cousiño, actualmente llamado Parque O'Higgins, adquiriendo un nuevo significado, como el de preservar un sentido de la naturaleza en las ciudades.
Este aporte moderno se constituye así en una extraordinaria plataforma -un espacio público inclusivo- al servicio del reconocimiento y el desarrollo de la tan necesaria integración social. Ahora bien, si observamos los metros cuadrados de áreas verdes -plazas y parques- por habitante en las diferentes comunas, se podrá comprobar que existen sectores de la población para quienes la idea de este tipo de espacio público simplemente no existe ${ }^{11}$. ¿Es posible extrañarse si los escasos parques urbanos -en el caso de Santiago- sufren percances debido al mal uso de ellos, por ejemplo, con motivo del desarrollo de eventos masivos? Un observador externo podría afirmar sin equivocarse que el espacio público no existe en Chile, en tanto lugar de encuentro para construir "sociedad" -en la acepción más completa del término-, congregando a seres sociales ${ }^{12}$.

\section{Usos, funciones, tipos y calidad del espacio público}

Las primeras tres acepciones, el uso (empleo continuado y habitual de algo), la función (tarea que corresponde realizar a una entidad o persona) y el tipo (modelo representativo de algo) son categorías que, en la práctica, no necesariamente convergen hacia una misma definición. A pesar de ello, hemos querido agruparlas pues nos permiten relevar ciertas ambigüedades que se presentan en la

Solo el $26,3 \%$ de los condominios sociales realizados en la ciudad de Santiago en los últimos 77 años tienen buena accesibilidad a áreas verdes (Valdivia 2014)

12 La desigualdad expresada en áreas verdes: comunas con población acomodada tienen más de $10 \mathrm{~m}^{2}$ por habitante (Vitacura 21,8; Providencia 14,3; La Reina 12,1; Las Condes $10,1)$ versus comunas con población vulnerable que bordean los $2 \mathrm{~m}^{2}$ por habitante (Independencia 1,3; La Cisterna 1,6; Pudahuel y El Bosque 1,9; Puente Alto 2,2) (Rojas 2013). 
percepción de los espacios públicos, toda vez que, pudiendo ser concebidos de una manera para un cierto uso, pueden adquirir cierta carga simbólica -por razones a veces difíciles de identificar- que los hace acoger una cierta función ritual en momentos que sería propio acogerlos en espacios de diferente tipo. La explicación podría encontrarse, justamente, en la carencia de espacios públicos apropiados para las diferentes expresiones sociales contemporáneas.

Como ejemplo está la Plaza Italia o Plaza Baquedano ${ }^{13}$, cuya denominación común social está referida a la existencia de alguno de los monumentos allí existentes, y cuyo diseño obedece a una articulación del encuentro de vías y parques. No obstante, en fechas y horas específicas se constituye en el espacio de una concentración espontánea, mayoritariamente de actos de celebración de triunfos deportivos, donde se congregan desde sus diferentes barrios, transversalmente, distintos grupos sociales, y se vive una suerte de euforia gregaria colectiva.

En cuanto al uso, el espacio público es el soporte físico, el escenario cotidiano, de interacciones sociales urbanas colectivas. Se caracteriza físicamente por su accesibilidad, sin embargo, es necesario diferenciarlo del espacio privado con apariencia de espacio público, como son, por ejemplo, los modernos centros comerciales.

Desde el punto de vista de la función que cumple, es posible señalar al menos tres: la función social, la función cultural y la función política:

13 Plaza ubicada en la ciudad de Santiago, capital metropolitana de Chile.
- En lo social es el espacio que permite el contacto entre las personas; aporta al proceso de construcción de identidad de los individuos, mediante la visibilización y la comparación social del sujeto a nivel intragrupal y exo-grupal a partir de la pertenencia y el uso del espacio. A nivel intra-grupal el sujeto tiende a buscar semejanzas con los miembros del mismo grupo de pertenencia, y en la comparación con el exo-grupo tiende a buscar elementos que le permitan diferenciarse, reforzando positivamente la identidad colectiva personal y grupal.

- En lo cultural permite y sustenta la vida urbana, y el desarrollo de la urbanidad como manifestación de ella. Esta última se define como el adecuado y correcto comportamiento expresado con modales que demuestren respeto hacia los demás, cuestión coherente con la norma cultural imperante en dicho grupo social.

- En lo político es el espacio donde pueden y deben expresarse y manifestarse las diferentes visiones existentes en el seno de una comunidad.

Otra manera de establecer funcionalidades en el espacio público es poniendo el acento en la mayor o menor necesidad que tenemos de hacer actividades en él, lo que determina, además, un tratamiento muy diferenciado, tanto del espacio propiamente tal, como del contexto urbano donde éstas se llevan a cabo. Así, podemos identificar la existencia de actividades necesarias, actividades opcionales y actividades sociales (Gehl 2006).

En lo necesario, un buen ejemplo lo constituye el desplazarse de un punto a otro de la ciudad para atender tareas cotidianas como trabajar, estudiar, hacer gestiones, etc. En las actividades 
opcionales, ejemplos característicos los constituyen acciones como sentarse a tomar el sol, a mirar el paisaje, a leer, etc. En el ámbito de las actividades sociales, destaca la concurrencia a actividades masivas como conciertos $u$ otras en espacios específicos muy propias de la adolescencia-, donde se espera tanto poder visibilizar como visibilizarse en búsqueda de la interacción con otros por intereses comunes.

En cuanto al tipo de espacios públicos existentes, los espacios públicos podrían agruparse, actualmente, en función de sus características, las que podrían clasificarse como funcionales, económicas y culturales. A saber:

- La vinculación comprende por regla general los espacios destinados a las vías de circulación abiertas, es decir, desde las calles a las carreteras y las vías férreas, y que pretenden vincular a unos con otros de acuerdo a los intereses o necesidades del grupo humano.

- El intercambio de bienes y servicios comprende las ferias, los mercados, los centros comerciales, como también los espacios reservados a la prestación de servicios, como escuelas, hospitales, edificios de la administración pública estatal o local, etc., todos con funciones distintas pero que tienden a congregarse.

- El desarrollo cultural comprende desde las bibliotecas, teatros y cines, como también los espacios de congregación trascendente y simbólica, que abarcan tanto la esfera religiosa como laica, santuarios y explanadas para la conmemoración histórica, hasta las plazas y los parques, que permiten el esparcimiento de la población y la contemplación de la naturaleza, todo esto con el propósito de permitir su integración, desarrollo y socialización con otros individuos.

También se pueden observar los espacios públicos a partir de la calidad de las relaciones sociales que facilitan, por ser espacios que posibilitan la vida cotidiana de los distintos actores sociales simultáneamente, construyendo identidad a partir de los diferentes universos simbólicos que se expresan y que, potencialmente, podrían integrarse. El espacio público supone uso social a nivel individual y colectivo, cuestión que se concreta en la experiencia de diversas actividades características, entre las que existe gran cantidad de alternativas. De acuerdo al uso social colectivo, los espacios públicos han de permitir la expresión colectiva, las manifestaciones cívicas, la visibilidad de los diversos grupos sociales, tanto a escala de barrios como de la centralidad urbana.

De acuerdo con lo anterior, se puede destacar como característica primordial del espacio público su capacidad de albergar al ciudadano para que exprese y desarrolle su dimensión social como sujeto de derecho, contribuyendo a la construcción de mundo, articulando la subjetividad arraigada en el mundo privado con la intersubjetividad enraizada en lo público. En esta dicotomía privado-público, la persona se comunica más allá de lo privado y más allá de lo social, y se integra mediante la intersubjetividad.

No obstante su relevancia, el espacio público no siempre ha sido bien considerado como escenario para estudiar o comprender las relaciones interpersonales. Al contrario, la tendencia ha sido concebir que las relaciones 
interpersonales "reales" se dan en los espacios privados, donde es más frecuente constatar la vinculación directa con los otros, y conocer sus variaciones y los elementos que lo componen.

\section{Conclusiones}

El espacio público se concibe como aquél que permite la construcción de identidades y de realidades individuales compartidas que favorecen, o cuando menos, influyen en la integración y lacohesión social. Así, si se considera el incremento del proceso de urbanización acelerado que acontece a nivel planetario, y la complejidad y la importancia de la situación de los espacios públicos como escenarios de encuentro y socialización, sobre todo atendiendo el incremento del malestar psicológico en la población urbana y lo que sucede con las relaciones sociales e interpersonales en las urbes contemporáneas -particularmente en América Latina $^{14}{ }^{14}$, se torna imprescindible advertir que el abordaje de su diseño, para ser eficaz, debe tener un carácter interdisciplinario.

En este sentido, se vuelve relevante tener la mayor claridad posible respecto de los factores que convergen en este complejo fenómeno. De allí que resulte pertinente apuntar al conocimiento y a la optimización de los elementos promotores del cambio deseado que, en definitiva, apuntan a una mayor y mejor calidad de vida, relaciones sociales e interpersonales que permitan la integración

14 La carencia de espacios públicos, la concentración urbana y la violencia en América Latina parecen ir aparejadas. "La mayoría de los países de la región tienen tasas de homicidio con niveles de epidemia, según la clasificación de la Organización Mundial de la Salud, con tasas mucho más altas que en otras regiones" (Programa de Naciones Unidas para el Desarrollo 2013: en línea). social de los habitantes de las urbes. Para ello es necesario, al menos, asumir dos cuestiones que resultan fundamentales:

- La concurrencia de aquellos actores y disciplinas encargadas del estudio tanto del comportamiento humano en el espacio público, como de las disciplinas encargadas de su diseño y materialización.

- Desarrollar un modelo que permita abordar el estudio, el diseño y la construcción de espacios públicos para el bienestar, la calidad de vida y la integración social.

En esta dirección, al considerar la información presentada y los aportes teóricos tratados en este artículo, resulta necesario dimensionar los factores que inciden tanto en el detrimento como en la mejora de la calidad de vida de las personas. Dicho análisis debe contener las condiciones de vida, las apreciaciones personales $y$, por cierto, los elementos culturales y subjetivos.

Para poder abordar la multiplicidad de factores señalados, y llegar a tener los elementos de contexto que permitan comprender una situación urbana específica donde se desarrollen actividades -optativas o socialesque posibiliten la integración social, es fundamental realizar investigación de campo. Estas investigaciones ameritan una mirada interdisciplinaria y el empleo del enfoque émico en su epistemología. Así, lo émico corresponde al enfoque de estudio "desde dentro" del sistema que se desea comprender -lo propio de la cultura nativa-, en este caso, los usuarios del espacio público.

Lo anterior implica también -y quizás por sobre todo- una mirada política no exenta de 
voluntad, pues asumir la integración social tiene un costo que se logra a través de lo émico. El objetivo de lograr integración social y calidad de vida requiere transitar, en suma, un camino que construya un modelo de desarrollo con un enfoque émico-ético.

En este sentido, se valoriza la mirada ideográfica, la cual ayuda a profundizar en la comprensión de los espacios públicos como realidades socialmente construidas, dinámicas y con sentido, desde sus propios actores sociales. Lo anterior se aborda tanto a nivel social como individual, sin desestimar la influencia de las lógicas de poder e interés -sean o no institucionales- que intervienen en estas construcciones.

En esta línea, ya hace cuarenta años existían planteamientos que indicaban que las personas encargadas de la planificación urbana debían considerar los antecedentes teóricos y técnicos provenientes de la psicología ambiental, para comprender tanto las conductas como las cogniciones humanas frente a los espacios habitados (Mercado y Covarrubias 1979). La propuesta, por tanto, no es del todo reciente pero sí amplifica la mirada interdisciplinaria para considerar distintas perspectivas en virtud de la subjetividad y la intersubjetividad de las personas. Es particularmente émica, ya que mira la construcción de los espacios públicos desde la construcción social de la realidad, proponiendo para ello apoyarse en metodologías participativas como las que aporta la psicología social aplicada y la antropología.

Desde esta perspectiva, es central no abordar a los usuarios como meros beneficiarios o entes pasivos, sino en su condición de ciudadanos capaces de ejercer una participación activa desde los inicios de un proyecto de desarrollo urbano, sobre todo de los requerimientos, los diagnósticos sociales y ambientales involucrados, el diseño de los espacios públicos, su potencial injerencia en las intervenciones, y en la etapas sucesivas de análisis y evaluación, hasta el uso mismo de dichos espacios y su conformación -y confirmación- como lugares con sentido de pertenencia e identidad. Para lograr esto es deseable que el espacio público pueda ser reconocido como tal mediante el uso de los miembros de una comunidad dada, es decir, debe hacerse inteligible al momento de su percepción, y así ser utilizado sin necesidad de que exista una explicación para ello.

En definitiva, destacamos la prioridad y la factibilidad de hacerse cargo, desde el diseño interdisciplinario de los espacios públicos urbanos, del problema contemporáneo asociado al malestar en las urbes. Para ello deben considerarse factores psicológicos, sociales, culturales, urbanísticos y políticos, que desempeñen un papel decisivo en la configuración de las realidades subjetivas y sociales, y de la calidad de vida en las ciudades. 


\section{Bibliografía}

Barrientos, J. 2005. Calidad de vida, bienestar subjetivo: Una mirada psicosocial. Santiago: Universidad Diego Portales.

Berger, P. y Luckmann, T. 2001. La construcción social de la realidad. Buenos Aires: Amorrortu Editores.

Botero, C. 2009. "La noción de contexto en la psicología ambiental de orientación transaccional". Cuadernos Hispanoamericanos de Psicología 10 (1): 5365. En línea, disponible en: http://www.uelbosque.edu. $\mathrm{co/sites/default/files/publicaciones/revistas/cuadernos}$ hispanoamericanos_psicologia/volumen10_numero1/ articulo_4.pdf (visitado el 13 de marzo del 2014).

Candia, C. 2001. "Seguridad ciudadana y estado policíaco". Polis: Revista Académica de la Universidad Bolivariana 1 (2): 1-6. En línea, disponible en: http://polis. revues.org/7944 (visitado el 13 de marzo del 2014).

Casey, E. S. 1998. The fate of place. A philosophical history. California: University of California Press.

De Jong Gierveld, J. y Van Tilburg, T. 2006. "A 6-Item scale for overall, emotional, and social loneliness. Confirmatory tests on survey data". Research on Aging 28 (5): 582-598. En línea, disponible en: http://roa.sagepub. com (visitado el 10 de enero de 2014).

Fernández-Ramírez, B. y Vidal, T. (Eds.). 2008. Psicología de la ciudad. Debate sobre el espacio urbano. Barcelona: Editorial UOC.

Fuentes, P. y Lawner, M. 2013. Memorias de un arquitecto obstinado. Concepción: Ediciones Universidad del Biobío.

Fundación Paz Ciudadana. 2013. Balance de la delincuencia 2012. En línea, disponible en: http:// www.pazciudadana.cl/publicacion/balance-de-ladelincuencia-2012/ (visitado el 13 de marzo del 2014).

Gagin, F. 2002. "Lo público y lo privado en los filósofos griegos. La cuestión de la libertad". Praxis Filosófica 15. En línea, disponible en: http://praxis.univalle.edu.co/ numeros/n15/francois_gagin.pdf (visitado el 20 de abril de 2015).

Gehl, J. 2006. La humanización del espacio urbano. Barcelona: Editorial Reverté.

Global Health Observatory. 2013. Urban population growth. Ginebra: World Health Organization. En línea, disponible en: http://www.who.int/gho/urban_health/ situation_trends/urban_population_growth_text/en/ (visitado el 13 de marzo del 2014).

Javaloy, F., Páez, D. y Rodríguez, Á. 2008. "Felicidad y relaciones interpersonales". Emociones positivas. Fernández, E. (Coord.). Madrid: Pirámide. 277-305.
Laboratorio BMW Guggenheim. 2013. 100 tendencias urbanas. En línea, disponible en: http://www. bmwguggenheimlab.org/100urbantrends/?v=2\#!/about/ (visitado el 13 de marzo del 2014).

Neira, H. 1997. "La urbe como espacio infeliz". Cuadernos Salmantinos de Filosofía XXIV. En línea, disponible en: http://neira.cl/publicaciones/118mestizaje-y-canibalismo-completo.html (visitado el 13 de marzo del 2014).

Mayell, H. 2010. "Documentary redraes humans' family tree". National Geographic News. En línea, disponible en: http://news.nationalgeographic.com/ news/2002/12/1212_021213_journeyofman.html (visitado el 13 de marzo del 2014).

Mercado, S. y Covarrubias, J. 1979. "La reacción humana ante la complejidad arquitectónica". Cuadernos de Comunicación 4 (47): 15-21.

Morris, D. 2000. Masculino y femenino. Barcelona: Plaza \& Janés Editores.

Obaid, T. 2008. Estado de la población mundial 2008. Nueva York: Fondo de Población de las Naciones Unidas. En línea, disponible en: http://www.unfpa.org/webdav/ site/global/shared/documents/publications/2008/swp08_ spa.pdf (visitado el 13 de marzo del 2014).

Orellana, A. 2013. "Migración desde zonas rurales hacia capitales regionales avanza con fuerza en 20 años". Diario El Mercurio. En línea, disponible en http://impresa. elmercurio.com/Pages/NewsDetail.aspx?dt=2013-0623\&dtB=28-06 2013\%200:00:00\&Paginald=12\&bodyid=3 (visitado el 23 de junio del 2013).

Organización para la Cooperación y el Desarrollo Económico. 2013. Índice para una vida mejor. En línea, disponible en: http://www.oecdbetterlifeindex.org/es/ countries/chile-es/ (visitado el 13 de marzo del 2014).

Programa de las Naciones Unidas para el Desarrollo. 2013. Informe regional de desarrollo humano 2013-2014. En línea, disponible en: http://www.undp.org/content/ dam/rblac/img/IDH/IDH-AL\%20Informe\%20completo. pdf (visitado el 13 de marzo del 2014).

2012. Desarrollo humano en Chile: Bienestar subjetivo. El desafío de repensar el desarrollo. En línea, disponible en: http://www.pnud.cl/prensa/4. asp\#Informes_de_Desarrollo_Humano_en_Chile (visitado el 13 de marzo del 2014).

Rodríguez, M. J. 2012. "Representación social de la noción de felicidad: Un estudio transcultural en muestras calificadas de estudiantes y profesionales de las áreas de la educación y la salud en Chile e Italia". Tesis doctorales 
en Psicología. Compendio 2011. Capella, C., Cavieres, H., Fernández, A. M., López, C. y Rodríguez, M. J. (Autores). Santiago: Universidad de Chile. 145-175.

Rojas, L. 2013. Planificación urbana participativa. En línea, disponible en: http://www.ciudadycalidad.usach.cl/ sites/ciudadycalidad/files/loreto_rojas.pdf (visitado el 13 de marzo del 2014).

Rousseau, J. 1980. Du contrat social. Argelia: SNED.

Salcedo, M. y Caicedo, S. 2008. "El espacio público como objeto de estudio en las ciencias sociales y humanas". Revista Científica Guillermo de Ockham 6 (1): 99-115. En línea, disponible en: http://www.redalyc.org/ pdf/1053/105312257008.pdf (visitado el 13 de marzo de 2014).

Toffler, A. y Toffler, H. 1995. La creación de una nueva civilización. Barcelona: Plaza \& Janés Editores.

Valdés, C. y Errázuriz, P. 2012. "Salud mental en Chile: El pariente pobre del sistema de salud". Claves de Políticas Públicas 11. En línea, disponible en: http://www. politicaspublicas.udp.cl/media/publicaciones/archivos/350/ Salud_Mental_en_Chile_El_Pariente_Pobre_del_Sistema_ de_Salud.pdf (visitado el 13 de marzo del 2014).
Valdivia, S. 2014. "Análisis de las áreas metropolitanas del Gran Santiago y Valparaíso". Seminario 77 años de condominios sociales en Chile. En línea, disponible en: http://www.minvuciudadano.cl/2014/01/seminario-77anos-de-condominios-sociales-en-chile/ (visitado el 13 de marzo del 2014).

Valera, S. 1996. "Análisis de los aspectos simbólicos del espacio urbano. Perspectivas desde la psicología ambiental". Revista de Psicología Universitas Tarraconensis 18: 63-84. En línea, disponible en: http://www.ub.edu/ dppss/valera/1996_Tarraconensis.pdf (visitado el 13 de marzo del 2014).

Vásquez-Honorato, L. y Salazar-Martínez, B. 2010. "Arquitectura, vejez y calidad de vida. Satisfacción residencial y bienestar social". Journal of Behavior, Health \& Social Issues 2: 57-70. En línea, disponible en: http:// es.scribd.com/doc/94606225/Arquitectura-Vejez-yCalidad-de-Vida (visitado el 13 de marzo del 2014).

World Health Organization. 2013. Official records of the World Health Organization $N^{\circ}$ 2. En línea, disponible en: http://whqlibdoc.who.int/hist/official_records/2e.pdf (visitado el 13 de marzo del 2014). 